\title{
Understanding and Studying Internet Culture by the Tool of Internet Language
}

\author{
Fengshunyu Chen ${ }^{1, \dagger}$, Quanying $\mathrm{Li}^{2, *}{ }^{*}$, Haoqing $\mathrm{Yao}^{3, \dagger}$ \\ ${ }^{1}$ Hunan College of Foreign Studies, Changsha City, Hunan Province, China \\ ${ }^{2}$ Jiaying University, Meizhou City, Guangdong Province, China \\ ${ }^{3}$ ULink College, Guangzhou City, Guangdong Province, China \\ ${ }^{*}$ Corresponding author. Email: guanghua.ren@gecacademy.cn \\ Those authors contributed equally.
}

\begin{abstract}
Language is a tool for understanding culture. The internet culture studied in this paper, as a heated research cultural category, not only has an impact on the internet social space and on the real society. Some studies have recognized and put forward that while internet language is an important part of internet culture, it can also be used as a tool to better understand internet culture. After people acquire internet language, they communicate their emotions and thoughts through internet language, thus achieving a wide range of social emotional communication, which has also become the reason for the diversity of internet culture. The purpose of this paper is to explore the internet language for the understanding of internet culture. To achieve this goal, the paper collects the speeches of relevant objects on various social media, such as Facebook, Twitter and Instagram, and sort them into text analysis.
\end{abstract}

Keywords: Internet language, Internet Culture, Second Language Acquisition, Linguistics

\section{INTRODUCTION}

Internet slang is a social dialect that is influenced by culture in various fields through the Internet. It is also defined as a language variant that facilitates online communication, with variations in pronunciation, vocabulary, syntax and discourse [1]. They have their own virtual speech communities and a language code with their own vocabulary and grammar. Internet culture is becoming increasingly diverse and the content of online language as a not only an organic whole but is also considered a linguistic variation of internet culture, allowing new words to develop and change into a new social discourse through various colloquialisms, homophones and real-time social phenomena [2][3]. Therefore, internet language can not only be used as a sign phenomenon of social dialect and internet culture that people have acquired after acquiring the first language, but also can be a useful tool for understanding internet culture.

As a language variant with strong fluidity and plasticity, internet language can not only influence internet culture but also penetrate daily social communication. In a mostly networked society, internet language becomes more and more likely to impact and influence people's social consciousness. In the process of information transmission, internet language becomes more and more diversified through the dissemination of different classes and people with different cultural ideas, and gradually becomes a new social dialect. Such social dialect helps people expand social internet by establishing weak ties through group communication on the Internet and promotes quantitative changes in the spread scope and qualitative changes in individual emotions, thus forming social emotional public opinion [4]. This means that not only have people unknowingly picked up a social dialect, but the language has influenced internet culture through its dissemination and innovation.

Therefore, this paper investigates Internet language as another aspect of second language acquisition and in a view of psychology explores how Internet language is regarded as a social dialect (a form of internet culture) to understand Internet culture. This study will focus on these main points. First, to find out how the Internet language influences Internet culture. And how the teenagers learn the internet language. Finally, this study will analyse the extent to which the online language used has an influence and role in analysing young people's understanding of online culture. 


\section{THE RELATIONSHIP BETWEEN INTERNET LANGUAGE AND INTERNET CULTURE}

As a social dialect of various varieties of conventional languages, internet language also has the characteristics of conventional languages and the original concept of "language". Gary B. Palmer, an American linguistic anthropologist, believes that language is just a symbol with sound, and all symbols are based on imagery, which can reflect people's direct experience and feelings of physical properties and abstract concepts [5]. At the same time, he also believes that language can not only be used to express ready-made images provided by experience and world outlook, but also cause or create new images, indicating that there is a co-construction relationship between image and language [5]. According to the above characteristics, it can be inferred that internet language, as the most brand-new language variant in the contemporary century, not only has the above characteristics, but also shows the image of the youngest generation and is constantly innovating.

Like the conventional relationship between language and culture, internet language and internet culture are mutually influenced and inseparable. Malinowski points out in 1987 that "language is a part of the overall culture, but it is not a system of tools, but a part of a set of pronunciation customs and spiritual culture." [6]. So, it can be concluded that the vocabulary, semantic grammar and cognitive mode of internet language are constructed in internet culture, and internet language also can be used as the carrier of internet culture, reflects the inherent nature of internet culture. On the other hand, from the perspective of diachronic linguistics, internet language has a certain time nature, which can reflect the changes of internet culture and the phenomenon of internet society and unconsciously inherit the internet culture of the early and early generations [6]. According to the internet language in different periods, people can understand the changes of internet culture and reveal the values and views of internet culture in different periods.

In addition, from an academic point of view, teenagers as the youngest generation are the group that is most likely to change internet culture today. According to previous research and investigated, 14- to 20-year-olds are more active online than any other age group. Dr. Max j. Coppes, M.D., PhD, MBA conducts an online survey in 2019, the results depict that more than $90 \%$ of adolescents use social media, and about $75 \%$ of adolescents have at least one active social media profile before the age of 17 [7]. And a study from the Pew Research Center finds out that more than 50 percent of 13- to 17-year-olds go online several times a day [7]. The above survey shows that teenagers are more susceptible to the influence of internet terms and the current situation of daily use of internet terms is more obvious. In addition, with the development and changeability of "we media", teenagers are not only affected by the existing internet terms, but also can use internet media to create new internet terms to express the current social environment and living conditions. And Guy Merchant also believes that teenagers and young adults are the pioneers of these processes of change because they skillfully take advantage of the possibilities of digital technology through the use of mobile phone SMS, email, web pages and online chat rooms, has fundamentally changed the face of literacy in all media [8]. Therefore, to a large extent, teenagers are the most vulnerable to the influence of internet culture and the main group that changes internet culture the most, as well as the largest group that has acquired, applied and created internet language. Therefore, this study will explore the relationship between the internet language learned by the largest group of creators of internet language and internet culture.

\section{TEENAGERS AND INTERNET LANGUAGE ACQUISITION}

Some studies have showed that teenagers not only acquire internet language because of the internet culture involved in social internet media or the popular language used by people around them and also acquire internet language as an unconscious instinct. Second language acquisition researchers believe that the acquisition of a second language after the acquisition of the original mother tongue usually occurs during adolescence or later, which also corresponds to the age of the group with the most online activity [9]. Second language acquisition is usually guided or controlled. Teenagers consciously learn and absorb the internet terms they want to use in their field or in their interest and internalize the information for future use. According to the linguistic conclusion of Chomsky in 1959, human beings have a set of physiological functions in the brain or mind that are specifically responsible for language acquisition and the use of natural language [9] [10] [12]. Because of this talent, human beings can process language with abstract rules to produce structured expressions [12]. According to the conclusion above, after teenagers acquire internet language, they externalize their original internal language for the second time, and thus generate new words and sentences, and then turn into a new generation of internet language and internet jokes through their speeches and communication on social media.

\subsection{Internet language acquisition and second language vocabulary acquisition model}

Teenagers can associate internet language with their mother tongue and acquire the initial acquisition of internet language. According to Zhao Haiyong, Luo Shaoqian \& Xing Jiaxin, in the formal stage of the L2 word acquisition model, L2 words are learned only through forms, because new words can be acquired only 
if the meanings of L2 words are connected or similar to the meanings of their mother tongue counterparts [11]. However, some compound words in internet languages are synthesized by combining two or more words and taking part or all of them to form a new word, which can act as a word in both grammar and semantics. The pronunciation and spelling of these phrases are different from traditional languages to some extent. Take English for example: Microserf refers to people who are addicted to the Internet. The normal expression is "people who are addicted to the Internet". Focus on the native language corresponding words and use pointer to connect native words and second language words to split the word "microserf" into two parts to understand with the original English meaning: "Micro" refers to internet, "Serf" refers to slave. These two words together mean being a slave to the Internet, but they can be used metaphorically to describe People who are addicted to the Internet, and to a certain extent they can be described as addicted to the Internet. This shows that by using the corresponding words of their mother tongue to understand internet language, teenagers can be quickly understood and remembered when they understand the preliminary grammar rules of internet language [11].

After activating the corresponding connection between internet words and their mother tongue words, with the growth of teenagers' language learning experience, internet words are further connected with their mother tongue counterparts more closely. In this stage, the lexeme information of the mother tongue is copied into the morpheme of the second language, forming a dichotomous entry with the semantic and syntactic information of its mother tongue counterpart. Although in this stage, the connection between the dichotomous term and the concept representation of the second language is very weak. This is because the morpheme information of binary lexical items is copied from their mother tongue counterparts [11]. Take the Internet word "microserf" for another example: from the perspective of English grammar and meaning, "micro-" is an adjective usually used at the beginning of a word, and "serf" is a noun usually used as the object of description, so the combination of the two will form a word with descriptive or figurative meaning. However, it is obvious that "microserf" and other such internet words are created by the original sentence meaning and grammar of mother tongue. It takes a short time to understand and imitate such words, but it takes a certain amount of time and use to fully learn them. In a word, this process is very similar to the acquisition process in the first stage, but the difference is that at this stage, teenagers begin to learn to use internet language instead of their mother tongue to express the concepts and words they want to express, although they still need the help of the relevant words in their mother tongue to achieve this process [11].
When the acquisition stage of teenagers to the second language integration stage, they can directly use internet language subconsciously for thinking communication and communication. As their language learning experience increases, the semantic and syntactic descriptions of their native language counterparts are copied into the L2 lexical clauses, resulting in the formation of an entry containing the L2 lexical forms and the native language head [11]. For example, when they often want to express that a person is often addicted to the Internet, they do not use the sentence pattern of "people who are addicted to the Internet." instead, they directly and subconsciously convert to the mode of using internet language and use the concise way of "microserf" to express. Then applying the theory proposed by Zhao Haiyong, Luo Shaoxi and XingJiaxin [11]. So, this shows that in the process of teenagers acquiring internet language through second language acquisition, the formal representation of internet words as second language words is gradually stabilized, and the connection between internet words and the concept they want to express is gradually strengthened. Finally, they can get rid of the help of their mother tongue counterpart words. It has completed the transformation from vocabulary connection to ideological and conceptual adjustment, enabling teenagers to communicate with each other in a barrier-free and flexible way [11].

\section{CHANGES BROUGHT BY EMOTIONAL COMMUNICATION TO INTERNET CULTURE}

After teenagers acquire internet language, the emotional speeches they post through various social media websites contain some internet words, or the internet language they create contains their emotions and thoughts. In this sense, more people learn and create new internet language.

\subsection{The emotional communication led the internet language have a widespread}

The rise of the Internet has given teenagers more occasions and opportunities to express their emotions and ideas besides on campus. This communication behavior is called "emotional social sharing" -- an academic term in the field of psychology. Some psychologists have also proposed that this social sharing of emotions is universal, repetitive and repeated. Therefore, it can be inferred that the speech with internet language published by teenagers can be spread to a certain extent and affect the emotions and ideas of a considerable number of people. Sui Yan and Li Yan also believe that any information on the Internet is no longer controlled by the information source once it enters the communication channel, and the social sharing of emotions is no exception [4]. 
Internet language not only has a certain humorous nature and the function of spreading various cultures but can also truly convey the ideas of the new generation and can serve as a symbol to reflect the social status quo. From the perspective of social structure theory combined with emotion, it can cause a condensation effect in the process of communication, that is, to promote the emotional integration of the masses and form a new social public opinion. For example, one of the current buzzwords in China, "involution" can also be called a pair of coils. Some people compare "involution" to a conch that grows inward and compresses (conch), or to a top that must spin faster or fall immediately [13]. In fact, this description is quite consistent with the meaning in the current context: "an irrational vicious competition that reduces the overall happiness at the cost of eliminating competitors", which expresses people's dissatisfaction and mockery of this undesirable social phenomenon. As the emotional carrier of contemporary internet culture, this kind of vocabulary enables others to empathize with the parties or real-time events better, thus generating new social public opinion. In addition, Sui Yan and $\mathrm{Li}$ Yan also believe that social culture influences emotional expression, emotional expression in turn constructs social culture and people use internet language to express emotions and store emotions, which also forms a unique internet mass culture [4]. Then it can be learned, the internet language is quite so a social language variant, this with a nature of the connotation of the internet language became the mainstream of the internet culture to some extent, enables the internet culture and the social morals to clasp and make the internet culture share social emotions and learning the culture of moral values.

\subsection{The communication function of internet language}

As the analysis presented above, Internet languages are mostly created and spread by teenagers, mostly creating new words or giving new connotations to existing words through collage of forms and combination of meanings [4]. The internet language created by them combines the current rising cultural trend and language rules in line with the prevailing moral values to make up for and satisfy their inferiority and vanity of being noticed by many people. But it can be seen from the previous information that most people who are active on the Internet are teenagers, which means that the words of those young speakers can greatly arouse the empathy of many people, because most of them are at similar age or have similar experiences. Therefore, this not only achieves the purpose of the speaker, but also forms a popular social speech -- public opinion through the dissemination of a considerable number of people. This kind of speech will continue to spread and precipitate and build a new social culture. This effect is the precipitate effect. Even after a period has passed, the social emotions contained in internet language will remain in internet language for a long time. As Sui Yan and Li Yan refer to Collins: "Interactive rituals are linked in chains over time, with the result that the last interaction (through emotions and symbols) becomes the input for the next interaction; So emotional energy tends to build up over time [4]." Therefore, it can be known that internet language can bring some changes in the social nature of internet culture through the spread and resonance of groups.

\section{CONCLUSION}

This study sets out to analyze the relationship between teenagers' internet language and internet culture, to sum up the extent to which the nature of internet language has an impact on internet culture. This study has identified the internet language as a social dialect and an important part of internet culture, can be regarded as a second language for people to acquire and can also be used as the carrier of internet culture to convey its spiritual value. It can be seen from the previous survey data and the theories of second language acquisition that teenagers are the easiest group to acquire and innovate internet language. Their emotional or experiential internet speech spreads through the Internet, making internet language and internet culture constantly innovate together, and making the internet culture in the contemporary stage to a large extent express the thought and spiritual value of the youngest generation -teenagers. Therefore, in the case of the continuous spread of such internet speech, it can be inferred that the spiritual thoughts contained in contemporary internet culture will become the mainstream thoughts of modern society to a large extent, and some thoughts with traditional nature and religious nature become secularized. However, it should not be ignored that internet language can convey negative emotions as well as positive emotions, which affect the trend and nature of internet culture to a great extent. If the state of the real society cannot be maintained well, or there is no correct guidance for teenagers to learn what internet language and how to use it correctly, the appearance of the future internet culture is unimaginable. Therefore, the future study can combine the views of this study to explore how to correctly acquire and use internet language, so that the internet culture is based on positive values and moral norms, to promote the positive development of realistic social thoughts.

\section{REFERENCES}

[1] Coltelli, Laura, ed.Winged Words: American Indian Writers Speak[ M] .Lin- coln: U of Nebraska . 1990.

[2] Cao Wangru, 2010. [Internet Language in the Perspective of Sociolinguistics], Journal of Inne $r$ 
Mongolia Agricultural University (Social Science Edition), No.4 2010(Vo 1.12 Sum No .52).

[3] Google Books. 2021. Learning Language and Culture Via Public Internet Discussion Forums. [online] Available at: <https://books.google.at/books?hl=en\&lr=\&id=VC8 WDAAAQBAJ \&oi=fnd \&pg $=$ PR2 \&dq $=$ understandi ng+internet+language+and+culture \&ots $=$ rl-iWbeGV\&sig=VWIVAzMhZCS8-

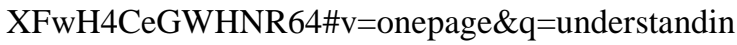
$\mathrm{g} \% 20$ internet $\% 20$ language $\% 20$ and $\% 20$ culture $\& \mathrm{f}=\mathrm{f}$ alse> [Accessed 15 October 2021].

[4] Sui Yan, Li Yan, 2020, [On the Role of the Internet Language in the Social Communication of Individual Emotions], Chinese Journal of Journalism \& Communication, ISSN : 1002-5685, No.01, 2020. pp.79-98.

[5] Palmer, G. B. Toward a Theory of Cultural Linguistics[M]. Austin: University of Texas Press, 1996.

[6] Gao Qingzhi, 2008, [On the relationship between language and culture], Anhui Literature (the second half of the month).

[7] Max J. Coppes, 2021. Teens and social media: When is it too much?. [online] University of Nevada, Reno School of Medicine. Available at: <https://med.unr.edu/news/archive/2019/coppesteens-and-social-media>. [Accessed 14 October 2021].

[8] Guy Merchant,2001, Teenagers in cyberspace: an investigation of language use and language change in internet chatrooms, Journal of Research in Reading, ISSN 0141-0423, Volume 24, Issue 3, 2001, pp 293-306.

[9] Patricia K. Kuhl, 2000, A new view of language acquisition, PNAS, vol. 97, no. 22, pp 11850-11857.

[10] Uni-due.de. 2021. Language acquisition. [online] Available at: <https://www.unidue.de/ELE/LanguageAcquisition.htm $>$ [Accessed 15 October 2021].

[11] Zhao Haiyong, Luo Shaoqian \& Xing Jiaxin, [Mental Lexicon and Second Language Vocabulary Acquisition Model], English Language Learning, ISSN: 1002-5553, No.04, 2018, pp60-64.

[12] Wei Xing, Yuan Fang, 2015. [Chomsky's internalism language view and its enlightenment on second language acquisition], Journal of Language and Literature Studies, No.05, pp 6-9.

[13] Clifford Geertz, Agricultural Involution: The Processes of Ecological Change in Indonesia,1960s. 